\title{
Die neue Euthanasie in Belgien - Wie steht es um die psychisch Kranken?
}

\author{
Euthanasia in Belgium - What about the Mentally III?
}

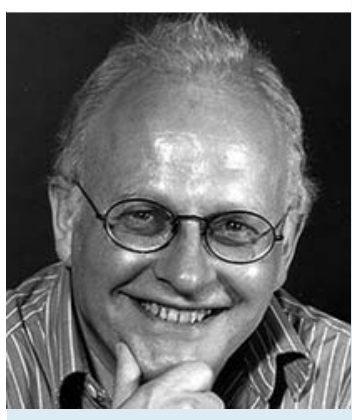

Asmus Finzen
Die „neue Euthanasie [1]“, die Tötung von kranken Menschen auf ihr eigenes Verlangen, ist in den Beneluxländern gesetzlich zugelassen. Sie wird seit mehr als einem Jahrzehnt - in den Niederlanden seit 20 Jahren - praktiziert - auch bei Kindern und Jugendlichen, auch bei psychisch Kranken. In Belgien nahmen zuletzt (2010/11) 2086 Menschen, davon 2,8\% psychisch Kranke [2], dieses Recht in Anspruch: das Recht, ihr eigenes Leben, das ihnen unerträglich geworden war, beenden zu lassen.

In den 3 Ländern herrscht weitgehender gesellschaftlicher Konsens, dass diese Praxis - in der dortigen Fachsprache als Euthanasie bezeichnet zu tolerieren ist. Deswegen werde ich den Begriff der Euthanasie in dieser Arbeit durchgehend für die Tötung auf Verlangen verwenden [3].

Die Außenperspektive ist eine andere: Verständnislosigkeit, vehemente Ablehnung und Furcht vor grenzüberschreitender Ansteckung. Dabei spielt die Angst vor einem „Dammbruch“ („Slippery Slope“) eine besondere Rolle. Dabei mag von besonderer Bedeutung sein, dass in 2 der 3 Länder die Tötung auf Verlangen und die Hilfe bei der Selbsttötung gemeinsam erfasst werden: auch die Hilfe beim Suizid gilt somit als Euthanasie. Die Angst vor dem Verlust der Grenzen spielt auch in der derzeitigen deutschen Debatte zur Beihilfe zum Suizid - v.a. auf der Seite der Gegner - eine zentrale Rolle. Der bekannte amerikanische Psychiater Herbert Hendin, damals Direktor der amerikanischen Suicide Foundation, veröffentlichte bereits 1997 [4] eine Monografie, in der er die wichtigsten Argumente gegen die neue Euthanasie zusammenfasste. Er sprach in diesem Zusammenhang von der „Dutch Cure“.

Die Verbindung von psychischer Krankheit und Euthanasie lässt uns Deutsche erschrecken. Psychisch Kranke und geistig Behinderte waren die Opfer der nationalsozialistischen Krankenmorde, die euphemistisch als Euthanasie bezeichnet wurden. Dabei war es auch dort angeblich zunächst darum gegangen, schwerst Kranken auf deren Ersuchen nach gründlicher Prüfung durch vertrauenswürdige Ärzte den „Gnadentod zu gewähren“ - so der Führererlass von 1939. Dass das der Auftakt zu einer der größten Massen-
mord-Aktionen der Geschichte war, konnten die Befürworter der Euthanasie, die es auch unter deutschen und deutschsprachigen Psychiatern gab, nicht wissen. Wenig bekannt ist beispielsweise die positive Haltung Eugen Bleulers zur Euthanasie schizophreniekranker Menschen, die er in einem Essay zur naturwissenschaftlichen Ethik [5] vertrat. Heute gibt es keine Entschuldigung dafür, nicht das Ende zu bedenken, wenn man sich für die Legalisierung der Tötung auf Verlangen einsetzt. Und die Behauptung/Annahme, dass sich ein staatlich verordneter Massenmord an Kranken und Behinderten nie wiederholen wird, zeugt von geschichtsvergessener Naivität.

Man kann sich mit einigem Recht die Frage stellen, wieso psychisch Kranke in den Beneluxländern überhaupt einbezogen werden. Dabei scheinen allerdings auch rechtliche Aspekte eine Rolle zu spielen: In der Schweiz ist beispielsweise ein Moratorium der Hilfe zur Selbsttötung bei psychisch Kranken, zu dem sich die Suizidhilfeorganisation Exit entschlossen hatte, durch einen Bundesgerichtsentscheid aufgehoben worden: Der Ausschluss von psychisch Kranken - allein wegen ihrer Krankheit - verstoße gegen das Antidiskriminierungsgesetz.

Über die Häufigkeit von Krankentötungen und Hilfen bei der Selbsttötung bei psychisch Kranken in diesen Ländern ist wenig bekannt. Jetzt ist ein Beitrag belgischer Wissenschaftlerinnen und Wissenschaftler im renommierten British Medical Journal erschienen [2], der dazu umfassende neue Erkenntnisse vermittelt. Die Untersuchung analysiert 100 aufeinanderfolgende Euthanasiegesuche belgischer psychisch Kranker aus den Jahren 2007-2011. Es handelt sich dabei um 77 Frauen und 23 Männer im Alter zwischen 21 und 80 Jahren (Median 47). Bei mehr als 90 dieser Kranken war mehr als eine psychiatrische Diagnose gestellt worden. Am häufigsten waren Depressionen (58) und Persönlichkeitsstörungen (50) diagnostiziert worden. Bei 12 wurde ein Asberger-Syndrom festgestellt. Bei 38 wurden vor einer Entscheidung weitere Untersuchungen und weitere Behandlungen verlangt. Im Ergebnis wurden 48 der 100 Euthanasiegesuche bewilligt und 35 ausgeführt. Von den übrigen „verschoben“ 8 
die Maßnahme aus eigenem Antrieb und 5 brachen sie ab. Sie begründeten das damit, dass allein die Option auf die Maßnahme ihnen so viel innere Kraft gegeben habe, dass sie weiterleben konnten. Ein Jahr nach Abschluss der Untersuchung (2012) waren 8 weitere Patienten tot, davon 6 durch Suizid, jeweils eine infolge terminaler Sedierung und Anorexia nervosa. Das ist die nüchterne Zusammenfassung der Daten. Dahinter stehen Leid und Verzweiflung. Außerdem bilden die Daten die Ergebnisse einer komplexen Prozedur ab, die ein Euthanasiegesuch in Belgien auslöst. Zwei der Patienten, die Suizid begangen hatten, begründeten das im Vorfeld mit der unerträglichen Länge des Verfahrens.

Das belgische Euthanasie-Gesetz von 2002 bestimmt die Voraussetzungen für die Bewilligung eines Euthanasiegesuchs explizit. Danach müssen die Antragsteller an „unerträglichen und unbehandelbaren körperlichen oder psychischen Störungen“ leiden. Die Subjektivität des „unerträglichen Leidens“ wird anerkannt. Demgegenüber sind die Merkmale der Unbehandelbarkeit aufgrund von medizinischen Kriterien festgelegt. Dabei können sowohl Gesuche von Kranken mit terminalen Leiden berücksichtigt werden, wie solche, deren Lebensende nicht unmittelbar bevorsteht. Menschen mit psychischen Krankheiten wurden im legislativen Verfahren erst nach langen Debatten und Expertenanhörungen einbezogen. Euthanasiegesuche müssen von selbstbestimmungsfähigen Personen schriftlich eingereicht werden. Diese werden von (s)einem Arzt überprüft. Wenn dieser die Voraussetzungen als erfüllt ansieht, müssen dessen Feststellungen von einem zweiten, unabhängig agierenden Arzt bestätigt werden. Wenn der natürliche Tod nicht in absehbarer Zeit zu erwarten ist, muss außerdem der Rat eines dritten Arztes eingeholt werden, der Psychiater sein sollte, alternativ Spezialist für die Krankheit des Gesuchsstellers. Wenn diese Voraussetzungen erfüllt sind, müssen der behandelnde Arzt und der Kranke gemeinsam zu dem Schluss kommen, dass kein anderer akzeptabler Weg zur Erleichterung des Leidens des Kranken vorstellbar ist. Wenn der zugezogene Psychiater die Auffassung vertritt, dass ein weiterer psychiatrischer Behandlungsversuch aussichtsreich sein könnte, darf das Gesuch nicht bewilligt werden. Wenn es positiv beschieden und die Euthanasie durchgeführt wird, muss das innerhalb von 4 Tagen an die dafür vorgesehene staatliche Aufsichtskommission gemeldet werden, die den Vorgang überprüft.

Der Weg zur Euthanasie in Belgien ist also keineswegs unkompliziert einfach, wie ausländische Kritiker das häufig unterstellen. Natürlich stellt sich die Frage, ob und wie umfassend die gesetzlichen Vorschriften in der Praxis eingehalten werden. Dies war nach Auffassung der Autoren der vorliegenden retrospektiven Untersuchung bei den 100 berücksichtigten Kranken der Fall. Zum praktischen Vorgehen haben die belgischen psychiatrischen Gesellschaften Leitlinien entwickelt, die ein 4-stufiges Vorgehen vorsehen.

1. Die gründliche Evaluation aller vorliegenden Befunde und der Krankengeschichte.

2. Diskussion alternativer Behandlungsmöglichkeiten einschließlich palliativmedizinischer Maßnahmen.

3. gründliche Aufklärung der Patienten über das Verfahren, über ihre Krankheit und deren Verlauf sowie eventueller Alternativen. Danach muss noch einmal überprüft werden, ob der Wille zu sterben eindeutig und entschieden geäußert wird.

4. Einbeziehung der Angehörigen. Gesetzlich vorgeschrieben ist die Auseinandersetzung mit den Angehörigen über die Todeswünsche des Patienten. Die Zustimmung der Angehörigen ist erwünscht aber nicht zwingend.
Wie oben erwähnt, hielten 35 der 48 Kranken, deren EuthanasieGesuch bewilligt worden war, an ihrer Entschlossenheit zu sterben fest. Die eigentliche Tötung wurde danach in 21 Fällen vom behandelnden Arzt, in den verbleibenden 14 Fällen von einem Arzt der Organisation LEIF durchgeführt, die einspringt, wenn ein behandelnder Arzt nicht zur Verfügung steht. Tötungsmittel war in allen Fällen Natrium-Thiopental. Tötungsort war in 28 Fällen die eigene häusliche Umgebung, in 5 Fällen die behandelnde Klinik und in jeweils einem Fall die Praxis des behandelnden Arztes und eine Palliativstation am Allgemeinkrankenhaus.

30 Patientinnen und Patienten starben in Gegenwart von Angehörigen oder Freunden, wie die Autoren hervorheben, in „angenehmer und positiver Umgebung“. Ebenso heben sie hervor, dass Patienten und Angehörige überzeugt waren, bei der Euthanasie handele sich um einen humaneren Tod als ein Tod durch Suizid. Sie erwarteten eine weniger schwierige Trauerperiode als das nach einem Suizid der Fall gewesen wäre. Die Autoren sprechen abschließend von einem „Drahtseilakt“. Auf der einen Seite sei ausreichend Zeit erforderlich, um die gesetzlichen und medizinischen Voraussetzungen für eine Tötung auf Verlangen zu klären. Auf der anderen Seite bestehe die Notwendigkeit, zu handeln, bevor der Patient sich wegen der Unerträglichkeit seines Leidens selber das Leben nehme. Diesen abschließenden Kommentaren ist zu entnehmen, dass die Autoren die Möglichkeit der Euthanasie in Belgien positiv sehen. Einzelne von ihnen sind, wie im Text angedeutet, auch selber bei solchen Maßnahmen tätig.

Die vorliegende Veröffentlichung gibt umfassenden Aufschluss über die Situation in Belgien, speziell einen Überblick über die Bedingungen der Einbeziehung psychisch Kranker in Euthanasiemaßnahmen. Sie ist auch wichtig, wenn man diese, wie der Verfasser, mit Nachdruck ablehnt. Für unsere derzeitige deutsche Diskussion ist sie gewiss nur beschränkt hilfreich $[6,7]$. Aber sie zeigt, wie gewaltig der Unterschied zwischen der Hilfe zur Selbsttötung und der Krankentötung ist, obwohl auch in der deutschen Debatte von interessierten Kreisen immer wieder versucht wird, diese Grenzen zu verwischen. Ganz ohne Zweifel ist die Tötung von kranken Menschen ebenso wenig eine ärztliche Aufgabe, wie die Hilfe bei der Selbsttötung. Aber während die eine in Deutschland illegal ist und auch nicht legalisiert werden soll, bleibt die andere im Extremfall eine Frage ans Gewissen des behandelnden Arztes.

\section{Literatur}

1 Finzen A. Die Anmaßung einer neuen Euthanasie. Frankfurter Allgemeine Zeitung 2000; 61: 52

2 Thienpont L, Verhofstadt $M$, Van Loon $T$ et al. Euthanasia requests, procedures and outcomes for 100 Belgian patients suffering from psychiatric disorders: a retrospective, descriptive study. BMJ Open 2015; 5: e007454

3 Finzen A. Die Sprache der Sterbehilfe. Sozialpsychiatrische Informationen 2015; 45: 39-40

4 Hendin $H$. Seduced by Death. Doctors, Patients and the Dutch Cure. New York, London: WW Norton; 1997

5 Bleuler E. Die naturwissenschaftlichen Grundlagen der Ethik. Schweizer Archiv für Neurologie und Psychiatrie 1936; 19: 177-206

6 Kruse A. Das Leben des demenzkranken Menschen bis zu seinem natürlichen Ende begleiten: Eine Alternative zum (ärztlich) assistierten Suizid. Kommentar zu Gather J, Vollmann J. „Die ärztlich assistierte Selbsttötung und advance care planning - Medizinethische Überlegungen zur Selbstbestimmung am Lebensende von Menschen mit Demenz“. Psychiat Prax 2014; 41: 392 - 393

7 Gather J, Vollmann J. Die ärztlich assistierte Selbsttötung und advance care planning - Medizinethische Überlegungen zur Selbstbestimmung am Lebensende von Menschen mit Demenz. Psychiat Prax 2014; 41: 385 - 391 